\title{
An approximation property with respect to an operator ideal
}

\author{
by
}

\author{
Juan Manuel Delgado (Sevilla) and CÁndido Piñeiro (Huelva)
}

\begin{abstract}
Given an operator ideal $\mathcal{A}$, we say that a Banach space $X$ has the approximation property with respect to $\mathcal{A}$ if $T$ belongs to $\overline{\{S \circ T: S \in \mathcal{F}(X)\}^{\tau_{c}}}$ for every Banach space $Y$ and every $T \in \mathcal{A}(Y, X), \tau_{c}$ being the topology of uniform convergence on compact sets. We present several characterizations of this type of approximation property. It is shown that some of the existing approximation properties in the literature may be included in this setting.
\end{abstract}

1. Introduction. It is well known that a Banach space $X$ has the approximation property (for short, AP) if and only if $T \in \overline{\{S \circ T: S \in \mathcal{F}(X)\}}^{\tau_{c}}$ for every Banach space $Y$ and every $T \in \mathcal{L}(Y, X)$, where $\mathcal{F}(X)$ denotes the space of all finite rank operators on $X$ and $\tau_{c}$ is the topology of uniform convergence on compact sets in $X$. Given an operator ideal $\mathcal{A}$, we may ask the following: For which Banach spaces $X$ do we have $T \in \overline{\{S \circ T: S \in \mathcal{F}(X)\}}^{\tau_{c}}$ for every Banach space $Y$ and every $T \in \mathcal{A}(Y, X)$ ? This question leads to the notion of approximation property depending on the operator ideal $\mathcal{A}$, which will be denoted by $\mathrm{AP}_{\mathcal{A}}$. This is a different approach compared with that by Reinov [17, 19], Grønbæk and Willis [9, Lissitsin, Mikkor and Oja [12] and, recently, Berrios and Botelho [2], which is already considered as classic.

Obviously, if $X$ has the $\mathrm{AP}$, then $X$ has the $\mathrm{AP}_{\mathcal{A}}$ for every operator ideal $\mathcal{A}$. The main result of this paper is Theorem 2.3 in which several characterizations of the $\mathrm{AP}_{\mathcal{A}}$ are established, some of them involving the notion of $\mathcal{A}$-compactness introduced by Carl and Stephani [4. As an application, it is proven that if $p \geq 1$ and $\mathcal{A}=\mathcal{K}_{p}$, the ideal of $p$-compact operators, then the $\mathrm{AP}_{\mathcal{A}}$ is the $p$-approximation property in the sense of Sinha and Karn [21]. As another example, we show that the approximation property of order $p(1 / 2 \leq p<1)$ in the sense of Reinov [16] is included in this setting for a suitable choice of operator ideal. Finally, a characterization of the $\mathrm{AP}_{\mathcal{A}}$ in terms of a trace condition is presented.

2010 Mathematics Subject Classification: Primary 46B28; Secondary 47L20, 46B50. Key words and phrases: approximation property, Banach ideal. 
Our notation is standard. A Banach space $X$ will be regarded as a subspace of its bidual $X^{* *}$ under the canonical embedding $i_{X}: X \rightarrow X^{* *}$. We denote the closed unit ball of $X$ by $B_{X}$. If $A$ is a set in $X$, we write $\operatorname{aco}(A)$ for its absolutely convex hull. Given an absolutely convex and compact set $A \subset X, \operatorname{span}(A)$ is denoted by $X_{A}$. This space is normed by the Minkowski functional of $A$,

$$
\rho_{A}(x)=\inf \{t>0: x \in t A\} .
$$

It is well known that $\left(X_{A}, \rho_{A}\right)$ is complete and $A$ is its closed unit ball. The canonical inclusion map from $X_{A}$ into $X$, denoted by $j_{A}$, is obviously compact.

For Banach spaces $X$ and $Y$, the Banach space of all bounded linear operators from $X$ to $Y$ is denoted by $\mathcal{L}(X, Y)$. In this space, we denote by $\tau_{c}$ the topology of uniform convergence on compact sets in $X$. If $\mathcal{A}$ is an operator ideal, then $\mathcal{A}^{d}$ denotes its dual operator ideal and $\mathcal{A}^{\text {sur }}$ the surjective hull of $\mathcal{A}$. We denote by $\mathcal{L}, \mathcal{K}$ and $\mathcal{F}$ the operator ideals of bounded, compact and finite rank linear operators, respectively. We also need the following operator ideals: $\mathcal{N}_{p}, p$-nuclear operators; $\mathcal{Q N}_{p}$, quasi $p$-nuclear operators; and $\Pi_{p}$, $p$-summing operators. We refer to Lindenstrauss-Tzafriri's book [1] for the classical approximation properties and to Pietsch's book 15] for operator ideals (see also Diestel, Jarchow and Tonge [8] for common operator ideals like $\mathcal{N}_{p}$ and $\Pi_{p}$, and Persson and Pietsch [14] for $\mathcal{Q} \mathcal{N}_{p}$ ).

\section{An approximation property with respect to an operator ideal}

Definition 2.1. We say that a Banach space $X$ has the approximation property with respect to the operator ideal $\mathcal{A}$ (for short, $\mathrm{AP}_{\mathcal{A}}$ ) if for every Banach space $Y$ and $T \in \mathcal{A}(Y, X)$, we have $T \in \overline{\{S \circ T: S \in \mathcal{F}(X)\}}{ }^{\tau_{c}}$.

It is well known that a Banach space $X$ has the AP if and only if the identity map on $X$ can be approximated by finite rank operators uniformly on compact subsets of $X$. In order to obtain an analogous result for the $\mathrm{AP}_{\mathcal{A}}$, we recall the notion of $\mathcal{A}$-compactness introduced by Carl and Stephani [4].

If $\mathcal{A}$ is an operator ideal and $X$ is a Banach space, a set $A \subset X$ is said to be $\mathcal{A}$-compact if there exist a Banach space $Z$, an operator $T \in \mathcal{A}(Z, X)$ and a compact set $K \subset Z$ such that $A \subset T(K)$. We denote by $\mathfrak{M}_{c}^{\mathcal{A}}(X)$ the family of all $\mathcal{A}$-compact subsets of $X$. Relying on this concept, the notion of $\mathcal{A}$-compact operator is defined in an obvious way: an operator $T \in \mathcal{L}(X, Y)$ is said to be $\mathcal{A}$-compact if $T\left(B_{X}\right)$ is $\mathcal{A}$-compact in $Y$. The set of all $\mathcal{A}$ compact operators between Banach spaces is denoted by $\mathcal{K}^{\mathcal{A}}$. For the sake of completeness, we list several properties of these concepts (see Sections 1 and 2 in [4] for more details): 
Proposition 2.2. Let $\mathcal{A}$ be an operator ideal and let $X$ be a Banach space. Then

(i) $\mathfrak{M}_{c}^{\mathcal{A}}(X)=\mathfrak{M}_{c}^{\mathcal{A}^{\text {sur }}}(X)$,

(ii) $\mathfrak{M}_{c}^{\mathcal{A}}(X)=\mathfrak{M}_{c}^{\mathcal{A} \circ \mathcal{K}}(X)$,

(iii) $\mathcal{K}^{\mathcal{A}}=\mathcal{A}^{\text {sur }} \circ \mathcal{K}$.

We are now ready to state our main result.

Theorem 2.3. Let $\mathcal{A}$ be an operator ideal and $X$ a Banach space. The following statements are equivalent:

(i) $X$ has the $\mathrm{AP}_{\mathcal{A}}$.

(ii) For every space $Y$ and $T \in \mathcal{A} \circ \mathcal{K}(Y, X), T \in \overline{\{S \circ T: S \in \mathcal{F}(X)\}}^{\tau_{c}}$.

(iii) For every space $Y$ and $T \in \mathcal{A} \circ \mathcal{K}(Y, X), T \in \overline{\{S \circ T: S \in \mathcal{F}(X)\}}^{\|\cdot\|}$.

(iv) For every $\varepsilon>0$ and $A \in \mathfrak{M}_{c}^{\mathcal{A}}(X)$, there exists $S \in \mathcal{F}(X)$ such that $\|S x-x\|<\varepsilon$ for all $x \in A$.

(v) For every space $Y, \mathcal{F}(Y, X)$ is $\tau_{c}$-dense in $\mathcal{A}^{\text {sur }}(Y, X)$.

(vi) For every space $Y, \mathcal{F}(Y, X)$ is $\|\cdot\|$-dense in $\mathcal{K}^{\mathcal{A}}(Y, X)$.

Proof. (ii) $\Rightarrow$ (ii). This is obvious.

(ii) $\Rightarrow$ (iii). Consider a Banach space $Y$ and $T \in \mathcal{A} \circ \mathcal{K}(Y, X)$. Bearing in mind the well known fact that $\mathcal{K}=\mathcal{K} \circ \mathcal{K}$, we can write $T=T_{1} \circ T_{2}$, where $T_{1} \in \mathcal{A} \circ \mathcal{K}(Z, X)$ and $T_{2} \in \mathcal{K}(Y, Z)$ for a certain Banach space $Z$. By hypothesis, given $\varepsilon>0$, there exists an operator $S \in \mathcal{F}(X)$ such that $\left\|S \circ T_{1} z-T_{1} z\right\|<\varepsilon$ for all $z \in T_{2}\left(B_{Y}\right)$ and this yields $\|S \circ T-T\|<\varepsilon$.

(iii) $\Rightarrow$ iv). This is immediate in virtue of Proposition 2.2(ii).

$(i \mathrm{iv}) \Rightarrow(\mathrm{v})$. This is easy on taking a glance at Proposition 2.2(i).

$(\mathrm{v}) \Rightarrow$ (vi). This follows easily using Proposition 2.2(iii).

vii) $\Rightarrow$ (i). Let us consider a Banach space $Y$, an operator $T \in \mathcal{A}(Y, X)$, a compact set $K \subset Y$ and $\varepsilon>0$. According to [20, Lemma 4.11], there exist two compact sets $D$ and $E$ in $Y$ satisfying $K \subset D \subset E$ and $D$ is compact in $Y_{E}$. If we put $F=T(D)$ and $G=T(E)$ then the operator $j_{G}: X_{G} \rightarrow X$ belongs to $\mathcal{K}^{\mathcal{A}}\left(X_{G}, X\right)$ since $j_{G}\left(B_{X_{G}}\right)=G$ is $\mathcal{A}$-compact in $X$. By hypothesis, there exists $R \in \mathcal{F}\left(X_{G}, X\right)$ so that $\left\|R-j_{G}\right\|<\varepsilon / 2$. The operator $R$ admits a representation of the form $R=\sum_{k=1}^{n} \psi_{k} \otimes x_{k}$ with $\psi_{k} \in\left(X_{G}\right)^{*}$ and $x_{k} \in X$. However, the map $j_{G}$ is injective so $\left(j_{G}\right)^{*}$ has dense image in $\left(X_{G}\right)^{*}$ for the topology of uniform convergence on compact sets in $X_{G}$. Hence, for each $k=1, \ldots, n$, there exists $x_{k}^{*} \in X^{*}$ such that

$$
\sup _{x \in F}\left|\left\langle\psi_{k}-\left(j_{G}\right)^{*}\left(x_{k}^{*}\right), x\right\rangle\right|<\frac{\varepsilon}{2 \sum_{k=1}^{n}\left\|x_{k}\right\|} .
$$

Put $S=\sum_{k=1}^{n} x_{k}^{*} \otimes x_{k}$ and notice that, for each $x \in T(K) \subset F$,

$$
\|S x-x\|=\left\|S \circ j_{G}(x)-j_{G}(x)\right\| \leq\left\|S \circ j_{G}(x)-R(x)\right\|+\left\|R(x)-j_{G}(x)\right\| .
$$

This yields $\|S \circ T y-T y\|<\varepsilon$ for all $y \in K$. 
If $\mathcal{A} \supset \mathcal{K}$, then $\mathfrak{M}_{c}^{\mathcal{A}}(X)$ is the class $\mathfrak{M}_{c}(X)$ of all relatively compact subsets of $X$. This is a consequence of Proposition 2.2 (ii):

$$
\mathfrak{M}_{c}(X)=\mathfrak{M}_{c}^{\mathcal{L}}(X)=\mathfrak{M}_{c}^{\mathcal{L} \circ \mathcal{K}}(X)=\mathfrak{M}_{c}^{\mathcal{K}}(X) \subset \mathfrak{M}_{c}^{\mathcal{A}}(X) .
$$

Thus, in view of Theorem 2.3 iv), we have:

COROLlary 2.4. If the operator ideal $\mathcal{A}$ contains the ideal of all compact operators, then the $\mathrm{AP}_{\mathcal{A}}$ is precisely the $\mathrm{AP}$.

In early 1980s, Reinov introduced the notion of the approximation property of order $p$ when $0<p \leq 1$ (see, e.g., [16]). A Banach space $X$ is said to have the approximation property of order $p$ (for short, $\mathrm{AP}_{p}$ ) if the restriction of $j: Y^{*} \hat{\otimes} X \rightarrow \mathcal{N}(Y, X)$ to the subspace of $Y^{*} \hat{\otimes} X$ consisting of all tensors $u=\sum_{n} y_{n}^{*} \otimes x_{n}, y_{n}^{*} \in Y^{*}, x_{n} \in X$, with $\sum_{n}\left(\left\|y_{n}^{*}\right\|\left\|x_{n}\right\|\right)^{p}<\infty$ is injective for all Banach spaces $Y$. Setting $q=p /(1-p)$, it was observed in [3] and [18] that $X$ has the $\mathrm{AP}_{p}$ if and only if the identity map on $X$ can be approximated by finite rank operators uniformly on Bourgain-Reinov $q$-compact subsets, that is, the subsets $A$ of $X$ for which there exists $\left(x_{n}\right) \in \ell_{q}(X)$ such that $A \subset\left\{\sum_{n} \alpha_{n} x_{n}:\left(\alpha_{n}\right) \in B_{\ell_{1}}\right\}=\overline{\operatorname{aco}}\left(x_{n}\right)$. Bourgain-Reinov compact sets and corresponding operators were recently studied in [1].

If $q \geq 1$ and $\mathcal{A}$ is the ideal consisting of all operators mapping bounded sets to Bourgain-Reinov $q$-compact sets, then $\mathfrak{M}_{c}^{\mathcal{A}}(X)$ is precisely the class of all Bourgain-Reinov $q$-compact sets in $X$. It is obvious that every subset belonging to $\mathfrak{M}_{c}^{\mathcal{A}}(X)$ is Bourgain-Reinov $q$-compact in $X$. For the converse, it suffices to bear in mind that, given a $q$-summable sequence $\left(x_{n}\right)$ in $X$, there exists a null sequence $\left(\delta_{n}\right) \searrow 0$ such that $\left(\delta_{n}^{-1} x_{n}\right)$ remains $q$-summable; hence, $\overline{\operatorname{aco}}\left(x_{n}\right) \subset T\left(\overline{\operatorname{aco}}\left(\delta_{n} x_{n}\right)\right)$, where $T \in \mathcal{A}\left(\ell_{1}, X\right)$ is defined by $T e_{n}=$ $\delta_{n}^{-1} x_{n}$. According to Theorem 2.3 (iv), we obtain:

Corollary 2.5. Let $p \in[1 / 2,1)$ and $q=p /(1-p)$. If $\mathcal{A}$ is the ideal of all operators mapping bounded sets to Bourgain-Reinov q-compact sets, then the $\mathrm{AP}_{\mathcal{A}}$ is precisely the $\mathrm{AP}_{p}$.

Let $1 \leq p<\infty$ and let $p^{\prime}$ be the conjugate index of $p$. A subset $A$ of $X$ is said to be relatively $p$-compact if there exists a $p$-summable sequence $\left(x_{n}\right)$ in $X$ such that $A \subset\left\{\sum_{n} \alpha_{n} x_{n}:\left(\alpha_{n}\right) \in B_{\ell_{p^{\prime}}}\right\}$. This notion appeared in [21] to introduce a sort of gradation of the approximation property: A Banach space $X$ has the p-approximation property if the identity map on $X$ can be approximated by finite rank operators uniformly on relatively $p$-compact subsets of $X$. Let us denote by $\mathcal{K}_{p}$ the ideal consisting of all $p$-compact operators (i.e., operators mapping bounded sets to relatively $p$-compact sets). According to [7, Proposition 3.11], $\mathcal{K}_{p}=\left(\mathcal{N}^{p}\right)^{\text {sur }}$, where

$\mathcal{N}^{p}(X, Y)=\left\{T \in \mathcal{L}(X, Y): T=\sum_{n} x_{n}^{*} \otimes y_{n},\left(x_{n}^{*}\right) \in \ell_{p^{\prime}}^{w}\left(X^{*}\right),\left(y_{n}\right) \in \ell_{p}(Y)\right\}$. 
Thus, $\mathcal{K}_{p}$ is a surjective ideal as also is $\Pi_{p}^{d}$ (being the dual of an injective ideal), so Theorem 2.3.v and [6, Theorem 2.1] yield

Corollary 2.6. Let $\mathcal{A}=\mathcal{K}_{p}, \Pi_{p}^{d}$ or $\mathcal{N}^{p}$. Then the $\mathrm{AP}_{\mathcal{A}}$ is precisely the p-approximation property.

In [21, Theorem 6.4], it is showed that every Banach space has the $p$ approximation property for $p \leq 2$. The following result follows directly from Theorem 2.3 $\mathrm{V}$ ) if we bear in mind the surjectivity of the ideal $\Gamma_{2}$ of 2factorable operators and the fact that every Hilbert space enjoys the AP.

Corollary 2.7. Every Banach space has the $\mathrm{AP}_{\mathcal{A}}$ whenever $\mathcal{A} \subset \Gamma_{2}$.

Remark 2.8. A direct consequence of Proposition 2.2(i) and Theorem 2.3 iv) is that $\mathrm{AP}_{\mathcal{A}} \equiv \mathrm{AP}_{\mathcal{A}^{\text {sur }}}$. From this, it is clear that (ii) and (iii) of Theorem 2.3 are also valid if $\mathcal{A} \circ \mathcal{K}$ is replaced with $\mathcal{K}^{\mathcal{A}}$. Nevertheless, $\mathcal{A}^{\text {sur }}$ cannot be replaced with $\mathcal{A}$ in Theorem 2.3(v). For an example, consider $\mathcal{A}=\mathcal{N}^{p}$. Then every Banach space $X$ has the property that $\mathcal{F}(Y, X)$ is $\|\cdot\|$-dense (thus, $\tau_{c}$-dense) in $\mathcal{N}^{p}(Y, X)$ for every space $Y$. But, if $p>2$, there are Banach spaces $X$ failing to have the $p$-approximation property [21, Theorem 6.7] so, according to Corollary 2.6, failing the $\mathrm{AP}_{\mathcal{N}^{p}}$. Bearing in mind that $\mathcal{N}^{p}=\mathcal{N}^{p} \circ \mathcal{K}$, this example also implies that $\mathcal{K}^{\mathcal{A}}$ cannot be replaced with $\mathcal{A} \circ \mathcal{K}$ in Theorem 2.3 vii).

Remark 2.9. Let $\left(\mathcal{A},\|\cdot\|_{\mathcal{A}}\right)$ be a Banach operator ideal. In [13], a Banach space $X$ is said to have the $\mathcal{A}$-AP if $\mathcal{F}(Y, X)$ is $\|\cdot\|_{\mathcal{A}}$-dense in $\mathcal{A}(Y, X)$ for all Banach spaces $Y$. The $\mathcal{A}$-AP and the $\mathrm{AP}_{\mathcal{A}}$ are different notions: as observed in [13, every Banach space has the $\mathcal{N}_{p}$-AP for all $p$; however, by Remark 2.8 . if $p>2$, there exist Banach spaces failing the $\operatorname{AP}_{\mathcal{N}_{p}}$.

Now, we present a specific characterization of the $\mathrm{AP}_{\mathcal{A}}$ for dual Banach spaces. The following lemma is needed.

Lemma 2.10. Let $\mathcal{A}$ be an operator ideal and $X$ and $Y$ Banach spaces. If $T \in \mathcal{K}^{\mathcal{A}}(X, Y)$, then $T^{* *} \in \mathcal{K}^{\mathcal{A}}\left(X^{* *}, Y\right)$.

Proof. Consider $T \in \mathcal{K}^{\mathcal{A}}(X, Y)$ and choose a Banach space $Z$, an operator $U \in \mathcal{A}(Z, Y)$ and a compact set $K \subset Z$ such that $T\left(B_{X}\right) \subset U(K)$. Then

$$
T^{* *}\left(B_{X^{* *}}\right)=T^{* *}\left({\overline{B_{X}}}^{\text {weak }^{*}}\right) \subset{\overline{T^{* *}\left(B_{X}\right)}}^{\text {weak }^{*}} \subset \overline{U(K)}{ }^{\text {weak }^{*}} .
$$

Since $U(K)$ is compact, it follows that $T^{* *}\left(B_{X^{* *}}\right) \subset U(K)$, and so we have $T^{* *} \in \mathcal{K}^{\mathcal{A}}\left(X^{* *}, Y\right)$.

Theorem 2.11. Let $\mathcal{A}$ be an operator ideal and $X$ a Banach space. The following statements are equivalent:

(i) $X^{*}$ has the $\mathrm{AP}_{\mathcal{A}}$. 
(ii) For every $\varepsilon>0$ and $A \in \mathfrak{M}_{c}^{\mathcal{A}}\left(X^{*}\right)$, there exists $S \in \mathcal{F}(X)$ such that $\left\|S^{*} x^{*}-x^{*}\right\|<\varepsilon$ for all $x^{*} \in A$.

(iii) For every space $Y$ and $T \in\left(\mathcal{K}^{\mathcal{A}}\right)^{d}(X, Y), T \in \overline{\{T \circ S: S \in \mathcal{F}(X)\}}\|\cdot\|$.

Proof. (i) $\Rightarrow$ (ii). This is immediate in view of Theorem 2.3 (iv) and bearing in mind the well known fact that the adjoints of finite rank operators

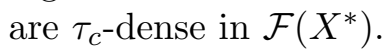

(ii) $\Rightarrow$ iii). Given $\varepsilon>0$ and $T \in\left(\mathcal{K}^{\mathcal{A}}\right)^{d}(X, Y)$, there exists $S \in \mathcal{F}(X)$ such that $\left\|S^{*} x^{*}-x^{*}\right\|<\varepsilon$ for all $x^{*} \in T^{*}\left(B_{Y^{*}}\right)$. Then $\|T \circ S-T\|=$ $\left\|S^{*} \circ T^{*}-T^{*}\right\|<\varepsilon$.

(iii) $\Rightarrow$ (i). By Theorem 2.3 (iii) and Remark 2.8, we are going to show that, for every Banach space $Y$ and $T \in \mathcal{K}^{\mathcal{A}}\left(Y, X^{*}\right), T \in \overline{\{S \circ T: S \in \mathcal{F}(X)\}}^{\|\cdot\|}$. So, fix $\varepsilon>0$ and $T \in \mathcal{K}^{\mathcal{A}}\left(Y, X^{*}\right)$. By Lemma 2.10 and the hypothesis, there exists an operator $S \in \mathcal{F}(X)$ so that $\left\|T^{*} \circ i_{X}-T^{*} \circ i_{X} \circ S\right\|<\varepsilon$. Thus, $\left\|T-S^{*} \circ T\right\| \leq\left\|i_{X}^{*} \circ T^{* *}-S^{*} \circ i_{X}^{*} \circ T^{* *}\right\|=\left\|\left(T^{*} \circ i_{X}\right)^{*}-S^{*} \circ\left(T^{*} \circ i_{X}\right)^{*}\right\|<\varepsilon$.

Corollary 2.12. If $p \leq 2$, every Banach space has the $\mathrm{AP}_{\mathcal{Q N}_{p}}$. On the other hand, if $p>2$, there exists a Banach space failing to have the $\mathrm{AP}_{\mathcal{Q N}_{p}}$.

Proof. Since $\mathcal{Q N}_{p} \subset \Gamma_{2}$ for every $p \leq 2$, the first assertion follows from Corollary 2.7. Now, if $p>2$, suppose, for contradiction, that every Banach space has the $\mathrm{AP}_{\mathcal{Q N}_{p}}$. According to Theorem 2.11, we have

$$
\left.\mathcal{K}_{\mathcal{Q} \mathcal{N}_{p}}^{d}(X, Y) \subset \overline{\mathcal{F}(X, Y)}\right)^{\|\cdot\|}
$$

for all Banach spaces $X$ and $Y$. On the other hand, from the equality $\mathcal{Q N} \mathcal{N}_{p}=$ $\mathcal{Q N}_{p} \circ \mathcal{K}$ [14, p. 32], it is clear that

$$
\mathcal{Q N}_{p}^{d} \subset\left(\mathcal{Q N}_{p} \circ \mathcal{K}\right)^{d} \subset\left(\left(\mathcal{Q N}_{p}\right)^{\text {sur }} \circ \mathcal{K}\right)^{d} .
$$

Finally, recall that $\mathcal{K}_{p}=\mathcal{Q N}_{p}^{d}[7$, Proposition 3.8]; so, in view of 2.1] and 2.2), we obtain $\mathcal{K}_{p}(X, Y) \subset \overline{\mathcal{F}(X, Y)} \|^{\|\cdot\|}$ for all Banach spaces $X$ and $Y$, contradicting [21, Theorem 6.7].

REMARK 2.13. Theorem 3.1 in 22$]$ states that $\mathcal{Q N} \mathcal{N}_{p}=\Pi_{p} \circ \mathcal{K}$. Hence, in virtue of Theorem 2.3 (iv) and Proposition 2.2 (ii), the above result is also valid if $\mathcal{Q N}_{p}$ is replaced with $\Pi_{p}$.

It is possible to characterize the $\mathrm{AP}_{\mathcal{A}}$ in terms of a trace condition. Let us consider in $\mathcal{L}(X, Y)$ the topology of uniform convergence on $\mathcal{A}$-compact sets in $X$. We denote this locally convex topology by $\tau_{c}(\mathcal{A})$. It is defined by the family of seminorms

$$
p_{A}(T)=\sup _{x \in A}\|T x\|,
$$

where $A$ is running over all the sets in $\mathfrak{M}_{c}^{\mathcal{A}}(X)$. The following result is immediate. 
Proposition 2.14. Let $\mathcal{A}$ be an operator ideal and $X$ a Banach space. The following statements are equivalent:

(i) $X$ has the $\mathrm{AP}_{\mathcal{A}}$.

(ii) For every space $Y$ and $T \in \mathcal{L}(X, Y), T \in \overline{\{T \circ S: S \in \mathcal{F}(X)\}}^{\tau_{c}(\mathcal{A})}$.

(iii) For every space $Y, \mathcal{F}(X, Y)$ is $\tau_{c}(\mathcal{A})$-dense in $\mathcal{L}(X, Y)$.

(iv) $\mathcal{F}(X)$ is $\tau_{c}(\mathcal{A})$-dense in $\mathcal{L}(X)$.

(v) For every space $Y$ and $T \in \mathcal{L}(Y, X), T \in \overline{\{S \circ T: S \in \mathcal{F}(X)\}}^{\tau_{c}(\mathcal{A})}$.

(vi) For every space $Y, \mathcal{F}(Y, X)$ is $\tau_{c}(\mathcal{A})$-dense in $\mathcal{L}(Y, X)$.

According to [4, Theorem 1.1], if $A \subset X$ is $\mathcal{A}$-compact, then $A \subset$ $\overline{\text { aco }}\left(x_{n}\right)$, where the sequence $\left(x_{n}\right)$ in $X$ is $\mathcal{A}$-convergent to zero (i.e., there exist an operator $S \in \mathcal{A}(Z, X)$ and a null sequence $\left(z_{n}\right)$ in $Z$ for which $\left.x_{n}=S z_{n}\right)$. Arguing as in the classical case, this property leads to the representation of every element $\Phi$ in $\left(\mathcal{L}(X, Y), \tau_{c}(\mathcal{A})\right)^{*}$ by

$$
\Phi(T)=\sum_{n}\left\langle y_{n}^{*}, T x_{n}\right\rangle
$$

for all $T \in \mathcal{L}(X, Y)$, where the sequence $\left(x_{n}\right)$ in $X$ is $\mathcal{A}$-convergent to zero and $\left(y_{n}^{*}\right) \in \ell_{1}\left(Y^{*}\right)$. Thus, we obtain

Proposition 2.15. Let $\mathcal{A}$ be an operator ideal and $X$ a Banach space. The following statements are equivalent:

(i) $X$ has the $\mathrm{AP}_{\mathcal{A}}$.

(ii) For every sequence $\left(x_{n}\right)$ in $X$ that is $\mathcal{A}$-convergent to zero, and every $\left(x_{n}^{*}\right) \in \ell_{1}\left(X^{*}\right)$ satisfying $\sum_{n}\left\langle x_{n}^{*}, x\right\rangle x_{n}=0$ for all $x \in X$, we have $\sum_{n}\left\langle x_{n}^{*}, x_{n}\right\rangle=0$.

Corollary 2.16. Let $\mathcal{A}$ be an operator ideal and $X$ a Banach space. If $X^{* *}$ has the $\mathrm{AP}_{\mathcal{A}}$, then so does $X$.

By Proposition 2.14, if $X$ has the $\operatorname{AP}_{\mathcal{A}}$ then $\mathcal{F}(Y, X)$ is $\tau_{c}(\mathcal{A})$-dense in $\mathcal{K}(Y, X)$ for every Banach space $Y$. In [5, Theorem 4.1] it is proved that the converse statement is true for the $p$-approximation property. The key to proving this result is the equality $\mathcal{K}_{p}=\mathcal{K} \circ \mathcal{K}_{p}$ [5, Theorem 3.1].

Proposition 2.17. Let $\mathcal{A}$ be an operator ideal satisfying $\mathcal{A}=\mathcal{K} \circ \mathcal{A}$. Then $X$ has the $\mathrm{AP}_{\mathcal{A}}$ if and only if $\mathcal{F}(Y, X)$ is $\tau_{c}(\mathcal{A})$-dense in $\mathcal{K}(Y, X)$ for every Banach space $Y$.

Proof. First we suppose $\mathcal{A}$ is a surjective ideal. To prove that $X$ has the $\mathrm{AP}_{\mathcal{A}}$, we will show that $\mathcal{F}(Y, X)$ is $\tau_{c}$-dense in $\mathcal{A}(Y, X)$ for every Banach space $Y$ (Theorem 2.3 v ) . Given $\varepsilon>0, T \in \mathcal{A}(Y, X)$ and a compact set $K \subset Y$, there exist a Banach space $Z$ and operators $T_{1} \in \mathcal{K}(Z, X)$ and $T_{2} \in \mathcal{A}(Y, Z)$ so that $T=T_{1} \circ T_{2}$. By hypothesis, there exists $S \in \mathcal{F}(Z, X)$ 
such that $\left\|S z-T_{1} z\right\|<\varepsilon$ for all $z \in T_{2}(K) \in \mathfrak{M}_{c}^{\mathcal{A}}(Z)$. Then we have $\left\|S \circ T_{2} y-T y\right\|<\varepsilon$ for all $y \in K$.

Now we consider an arbitrary operator ideal $\mathcal{A}$. Notice that

$$
\mathcal{A}^{\text {sur }}=(\mathcal{K} \circ \mathcal{A})^{\text {sur }}=\mathcal{K} \circ \mathcal{A}^{\text {sur }}
$$

where the last equality follows from the surjectivity of $\mathcal{K}$. Then the first part of the proof shows that $X$ has the $\operatorname{AP}_{\mathcal{A}^{\text {sur }}}\left(\equiv \mathrm{AP}_{\mathcal{A}}\right)$ if and only if $\mathcal{F}(Y, X)$ is $\tau_{c}\left(\mathcal{A}^{\text {sur }}\right)$-dense in $\mathcal{K}(Y, X)$ for every Banach space $Y$. Finally, just recall that $\tau_{c}(\mathcal{A})=\tau_{c}\left(\mathcal{A}^{\text {sur }}\right)$.

Acknowledgements. The authors are deeply grateful to the referee for calling their attention to the paper [4] in which $\mathcal{A}$-compactness is studied and for valuable suggestions that improved the paper substantially.

This research was supported by MTM2009-14483-C02-01 project (Spain).

\section{References}

[1] K. Ain, R. Lillemets and E. Oja, Compact operators which are defined by $\ell_{p}$-spaces, Quaest. Math. 35 (2012), 145-159.

[2] S. Berrios and G. Botelho, Approximation properties determined by operator ideals and approximability of homogeneous polynomials and holomorphic functions, Studia Math. 208 (2012), 97-116.

[3] J. Bourgain and O. Reinov, On the approximation properties for the space $H^{\infty}$, Math. Nachr. 122 (1985), 19-27.

[4] B. Carl and I. Stephani, On A-compact operators, generalized entropy numbers and entropy ideals, Math. Nachr. 119 (1984), 77-95.

[5] Y. S. Choi and J. M. Kim, The dual space of $\left(\mathcal{L}(X, Y), \tau_{p}\right)$ and the p-approximation property, J. Funct. Anal. 259 (2010), 2437-2454.

[6] J. M. Delgado, E. Oja, C. Piñeiro and E. Serrano, The p-approximation property in terms of density of finite rank operators, J. Math. Anal. Appl. 354 (2009), 159-164.

[7] J. M. Delgado, C. Piñeiro and E. Serrano, Operators whose adjoints are quasi p-nuclear, Studia Math. 197 (2010), 291-304.

[8] J. Diestel, H. Jarchow and A. Tonge, Absolutely Summing Operators, Cambridge Stud. Adv. Math. 43, Cambridge Univ. Press, Cambridge, 1995.

[9] N. Grønbæk and G. Willis, Approximate identities in Banach algebras of compact operators, Canad. Math. Bull. 36 (1993), 45-53.

[10] A. Grothendieck, Produits tensoriels topologiques et espaces nucléaires, Mem. Amer. Math. Soc. 16 (1955).

[11] J. Lindenstrauss and L. Tzafriri, Classical Banach Spaces I, Ergeb. Math. Grenzgeb. 92, Springer, Berlin, 1977.

[12] A. Lissitsin, K. Mikkor and E. Oja, Approximation properties defined by spaces of operators and approximability in operator topologies, Illinois J. Math. 52 (2008), 563-582.

[13] E. Oja, A remark on the approximation of p-compact operators by finite-rank operators, J. Math. Anal. Appl. 387 (2012), 949-952.

[14] A. Persson und A. Pietsch, p-nukleare und p-integrale Abbildungen in Banachräumen, Studia Math. 33 (1969), 19-62. 
[15] A. Pietsch, Operator Ideals, North-Holland, 1978.

[16] O. I. Reinov, Approximation properties of order $p$ and the existence of non-p-nuclear operators with p-nuclear second adjoints, Math. Nachr. 109 (1982), 125-134.

[17] O. I. Reinov, How bad can a Banach space with approximation property be?, Mat. Zametki 33 (1983), 833-846 (in Russian); English transl.: Math. Notes 33 (1983), 427-434.

[18] O. I. Reinov, A survey of some results in connection with Grothendieck approximation property, Math. Nachr. 119 (1984), 257-264.

[19] O. I. Reinov, How bad can a Banach space with approximation property be? II, J. Math. Sci. (New York) 112 (2002), 4065-4072.

[20] R. Ryan, Introduction to Tensor Products of Banach Spaces, Springer, London, 2002.

[21] D. P. Sinha and A. K. Karn, Compact operators whose adjoints factor through subspaces of $\ell_{p}$, Studia Math. 150 (2002), 17-33.

[22] D. P. Sinha and A. K. Karn, Compact operators which factor through subspaces of $\ell_{p}$, Math. Nachr. 281 (2008), 412-423.

Juan Manuel Delgado

Departamento de Matemática Aplicada I

Escuela Técnica Superior de Arquitectura

Avenida Reina Mercedes, 2

41012 Sevilla, Spain

E-mail: jmdelga@us.es
Cándido Piñeiro

Departamento de Matemáticas Facultad de Ciencias Experimentales Campus Universitario del Carmen 21071 Huelva, Spain E-mail: candido@uhu.es

Received May 28, 2012

Revised version March 10, 2013 
